\title{
GA-BP-based Microalgae Growth Prediction Model
}

\author{
Liu Jiaxing ${ }^{1+}$, Cui Shigang ${ }^{1}$, Chen miao ${ }^{1}$, Duan Huabiao ${ }^{1}$, Liu Yu ${ }^{1}$ \\ ${ }^{1}$ School of Automation and Electrical Engineering, Tianjin University of Technology and Education, China
}

\begin{abstract}
The microalgae growth model is difficult to construct. In order to predict the growth status of microalgae, the temperature and light intensity data of the microalgae growth are used as input signals, and the density and average radius of algae cells are used as pre measurement to build BP neural network prediction model. At the same time, to solve the problem that the BP weights and thresholds cannot be accurately obtained, resulting in unsatisfactory prediction accuracy, genetic algorithms (GA) were used to optimize the weights and thresholds of the BP neural network to improve the prediction ability of the BP. Prediction of the average radius of algal cells, GA-BP neural network has $13 \%$ and $10 \%$ higher decision coefficients $R^{2}$ than BP neural network, respectively. The BP neural network optimized by genetic algorithm has higher prediction accuracy. The research methods in this paper also provide new ideas for the study of microalgae growth models.
\end{abstract}

Keywords: Microallgae growth model, BP neural networks, Genetic algorithm, GA-BP.

\section{Introduction}

Microalgae, which have been around for billions of years, have high carbon dioxide absorption rates and high solar energy conversion rates[1]. From an economic point of view, microalgae can be described as microorganisms capable of capturing sunlight[2].

Microalgal biotechnology has been developed for a variety of commercial applications. Microalgae are photosynthetic organisms that can be used as food and in the pharmaceutical industry because some types of microalgae produce bioactive compounds such as antioxidants and antibiotics[3]. In addition, microalgae are rich in protein, vitamins and polysaccharides, making them a dietary supplement for human consumption[4]. Algae is also a promising renewable energy source. Microalgae have the potential to be used in biofuel production in an economically efficient and environmentally sustainable manner. The biofuel that is produced by research now has biology oil, biology hydrogen to wait[1]. In addition, certain species of microalgae have the ability to fix nitrogen and absorb heavy metals and phosphorus[5, 6]. Therefore, microalgae are also widely used in different kinds of sewage treatment, such as aquaculture sewage, municipal sewage, pharmaceutical wastewater, heavy metal wastewater, etc.[7]. There are many technological conditions that affect the growth of microalgae. Accurate model is very important to control and optimize the industrial production of algae biofuel[8]. Studies on algal growth models have looked at the effects of a single environmental factor, such as light and PH. Han et al. used a dynamic equation of light to describe the effect of light on the growth rate of algae[9]. Moheimani NR studied the effect of PH on the growth and lipid productivity of green algae. There are also studies on the effects of multiple factors[10]. Goldman, for example, introduced a microalgal growth multiplication model that took into account environmental factors such as light intensity, temperature, nutrition and $\mathrm{PH}[11]$. A good model should include all growth factors and the extent to which they affect growth, and should be able to predict the performance and productivity of a culture system under different operating conditions. This is difficult in theory, but even less so in practice[12]. Not only the complexity of the model, but also the difficulty of

\footnotetext{
+ Corresponding author. Tel.: + 13652139857.

E-mail address: 1171736029@qq.com
} 
parameter estimation are disadvantageous to the practical application. The parameters of the environment in the model also change over time and cannot be predicted in the long run, and the model needs to be rebuilt for different algae[13]. Neural network is widely used in forecasting[14]. BP neural network is the most widely used one. Light and temperature are the main factors that determine algal growth[15]. The object of this study was cymbidium oxysporum, one of the biological sources of the powerful antioxidant astaxanthin[16]. In the commercial production, two stages were used in the culture of cyanococcus aureus: the green stage of cell proliferation and growth, and the induction stage of astaxanthin accumulation[17]. In this study, aiming at the green stage of cell growth, BP neural network was established by using the light intensity and temperature data measured in experiments, as well as the algal cell density and the mean radius of algal cells under corresponding environmental conditions, and the BP neural network was trained with these data samples. The trained BP neural network is obtained and then optimized by genetic algorithm. Finally, the predicted results are compared.

\section{BP Neural Networks and Genetic Algorithms}

\subsection{BP Neural Networks}

BP(Back Propagation, BP)Neural networks have been widely used since they were introduced in 1986. They are systems that collect errors generated by a system, return them to the output, and use these errors to adjust the weights of neurons to improve the accuracy of the output[18].

BP network can save a large number of input/output mapping relations without writing out the relation equations of input and output. The BP network algorithm can theoretically express any input/output function. For BP network, a single hidden layer BP network can complete any $\mathrm{n}$ - to - $\mathrm{m}$ - dimensional mapping. The topology of BP neural network model includes input layer, hidden layer and output layer. See figure 1.

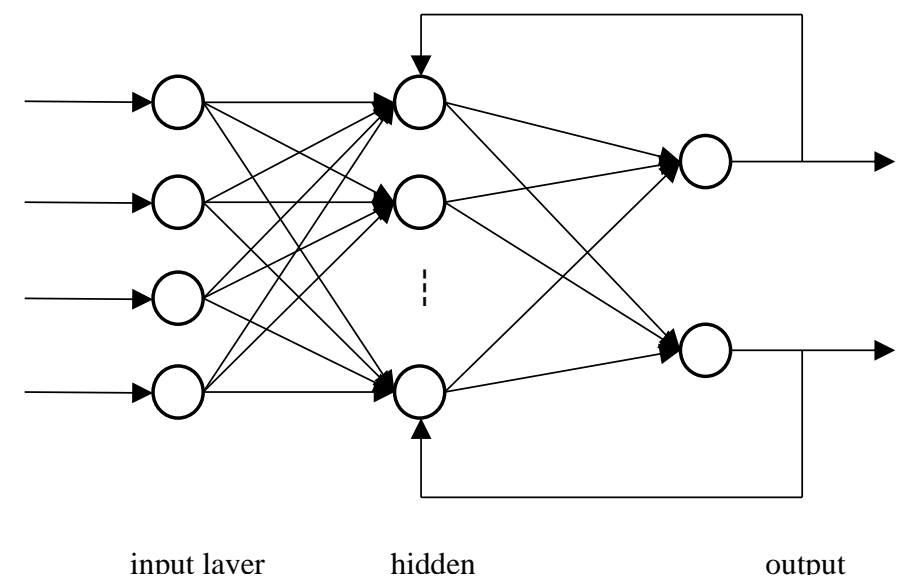

Fig. 1: BP neural network structure diagram

To construct BP network, it is mainly to set the input layer, output layer and hidden layer. In this study, the number of neurons in the input layer and the number of neurons in the output layer was 2 . It is difficult to determine the number of neurons in the hidden layer accurately. Here we draw on an empirical formula $n_{1}=$ $\sqrt{n+m}+a$, Where $\mathrm{m} \& \mathrm{n}$ are respectively the number of neurons in the output/input layer, a is the constant between ${ }^{[1,10]}$. Value of this experiment is $\mathrm{m}=2, n=2, a$ is $3, n_{1}=5$. The initial weights and thresholds of BP neural network have great influence on the training of the network, but they cannot be obtained accurately. The weight threshold of BP neural network can be handled well by genetic algorithm optimization. The training results are usually distinguished by the determination coefficient $R^{2}$, which determines the correlation between the simulated value and the observed value. The closer the value is to 1 , the better the training results are.

$$
R^{2}=1-\frac{\sum_{i=1}^{n}\left(Y_{p}-\overline{Y_{p}}\right)^{2}}{\sum_{i=1}^{n}\left(Y_{i}-\overline{Y_{l}}\right)^{2}}
$$

$Y_{p}$ is the simulation value, $\overline{Y_{p}}$ is the simulated average, $Y_{i}$ is the actual value, $\overline{Y_{l}}$ is the actual mean, $\mathrm{n}$ is the number of groups of data. 


\subsection{Genetic Algorithms}

Genetic Algorithm (GA) has made profound progress since it was first proposed in 1967 and has been applied in many fields. Mainly used in function optimization, machine learning, production scheduling and other problems[19]. This is an algorithm that simulates the "survival of the fittest" in nature. In application, the potential solution set of the problem is first coded, then the fitness function value of any possible solution is obtained, the fitness is sorted, and the appropriate solution is selected by the appropriate selection method. Then the selected solutions are cross-mutated to produce a new solution set. Repeat the above process to select the global optimal solution. The algorithm steps are shown in figure 2.

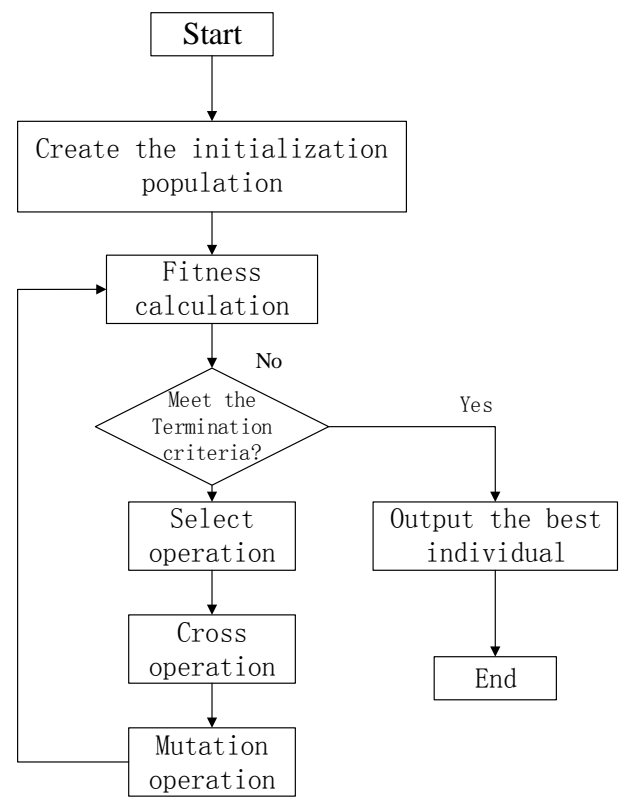

Fig. 2: Basic steps of genetic algorithm

\subsection{Genetic Algorithm to Optimize BP Neural Network}

The principle of BP neural network optimization based on genetic algorithm is to change the method of BP neural network using error back propagation to optimize weight and threshold, but to use genetic algorithm to optimize network weight threshold. The network is trained through experimental data. The inverse of the sum of squares of errors is taken as the fitness function, and the individual fitness function adopts the inverse of the absolute error sum of the predicted value and the actual value. The optimization process is shown in figure 3 .

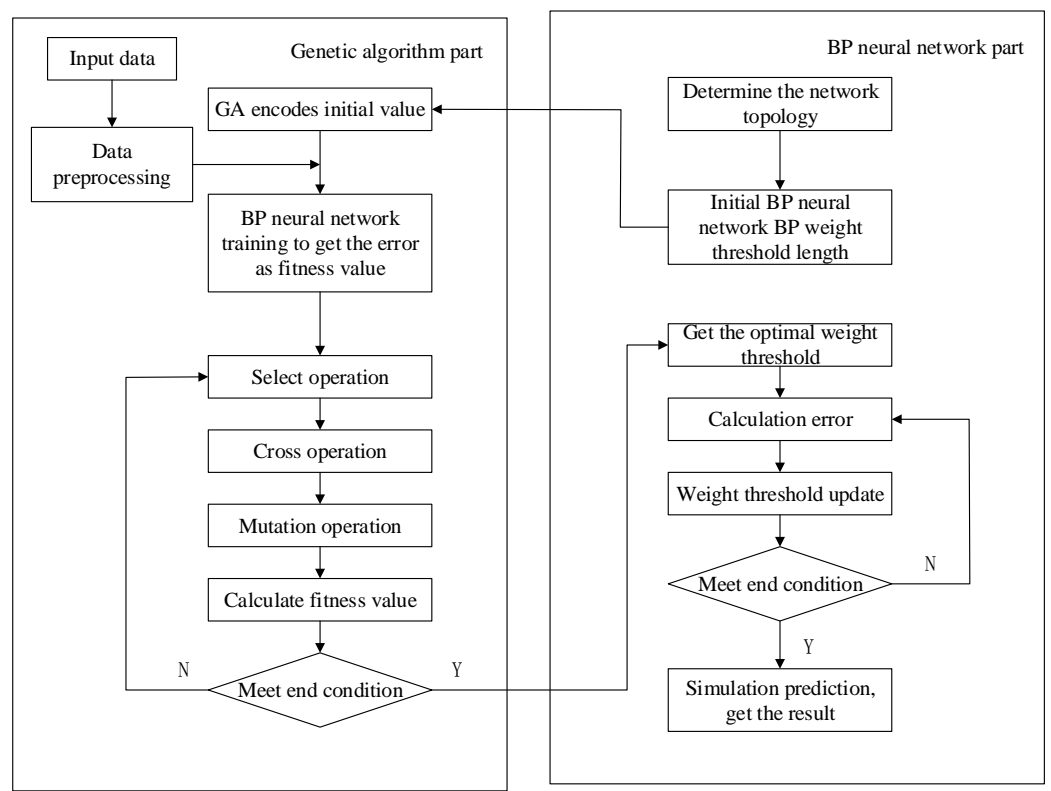

Fig. 3 : GA-BP neural network establishment process 


\section{Experimental Verification}

The freshwater algal species bank of institute of hydrobiology, Chinese academy of sciences was purchased. The algal liquid used in the green stage of cell growth was prepared by using BG11 medium as nutrient after the algal species were cultured for several times and reached the experimental dosage. In the experiment, the designed temperature of the light incubator was set as a temperature node at $4{ }^{\circ} \mathrm{C}$ per interval within the range of $16-32^{\circ} \mathrm{C}$, and the designed light source was set as a light intensity node at $5001 \mathrm{x}-50001 \mathrm{x}$ per interval, with a dark cycle of 12:12h. On the 8th day after culture, data collection was conducted in the ultra-clean workstation. The specific data collection methods use conventional microbial detection methods[20], a total of 50 groups of experimental data were measured.

In order to establish an algal growth prediction model, the experiment was divided into the following three steps: first, the input and output of the model were defined. The data were divided into the training group, the verification group and the test group according to 3:1:1 to prevent the neural network from overfitting, and the BP neural network was constructed. The second step is to use genetic algorithm to optimize the network. Finally, the optimized model is obtained, and the predicted value is obtained by inputting the test group data and comparing with the measured value.

Algorithm parameters were set, the number of hidden layer neurons was set as 5, the number of network iterations was set as 2500 , the expected error was 0.001 , and the learning rate was 0.05 . In the genetic algorithm part, the population size was set as 100 , the number of evolution was 60 , the crossover probability was 0.3 , and the mutation probability was 0.2 . After setting parameters and building a good training network, input the data of the test group. Table 1 shows the data of the test group and the predicted results. Fig. 4 and Fig. 5 show the comparison between the measured values of algal cell density and mean cell radius and the predicted results of ga-bp and BP neural network. Fig. 6 and Fig. 7 show the comparison of prediction errors of ga-bp and BP neural network for algal cell density and mean cell radius, respectively.

Table 1: Test group data and prediction results

\begin{tabular}{cccccccc}
\hline \multirow{2}{*}{$\begin{array}{c}\text { temperature } \\
\left({ }^{\circ} \mathrm{C}\right)\end{array}$} & $\begin{array}{c}\text { illumination } \\
(1 \mathrm{x})\end{array}$ & \multicolumn{2}{c}{$\begin{array}{c}\text { The actual measured } \\
\text { value }\end{array}$} & $\begin{array}{c}\text { GA-BP Algorithm } \\
\text { prediction }\end{array}$ & \multicolumn{2}{c}{ BP Algorithm prediction } \\
\cline { 3 - 8 }$\left(10^{4} / \mathrm{mL}\right)$ & $\begin{array}{c}\text { Mean } \\
\text { radius } \\
(\mu \mathrm{m})\end{array}$ & $\begin{array}{c}\text { Cell density } \\
\left(10^{4} / \mathrm{mL}\right)\end{array}$ & $\begin{array}{c}\text { Mean } \\
\text { radius } \\
(\mu \mathrm{m})\end{array}$ & $\begin{array}{c}\text { Cell density } \\
\left(10^{4} / \mathrm{mL}\right)\end{array}$ & $\begin{array}{c}\text { Mean } \\
\text { radius } \\
(\mu \mathrm{m})\end{array}$ \\
\hline 28 & 3500 & 24.673 & 8.297 & 24.529 & 8.169 & 25.819 & 9.863 \\
20 & 3000 & 17.456 & 11.327 & 17.512 & 11.597 & 16.019 & 10.654 \\
24 & 3000 & 19.727 & 20.498 & 19.781 & 20.39 & 17.283 & 19.124 \\
16 & 4500 & 9.386 & 18.945 & 9.937 & 18.545 & 11.397 & 19.895 \\
24 & 1500 & 14.437 & 17.341 & 14.312 & 17.543 & 13.427 & 17.007 \\
20 & 1000 & 10.754 & 9.874 & 10.951 & 9.764 & 8.896 & 10.654 \\
16 & 1000 & 8.063 & 21.257 & 8.379 & 21.468 & 6.063 & 22.547 \\
24 & 2500 & 19.328 & 22.897 & 19.007 & 23.007 & 18.029 & 21.543 \\
28 & 3000 & 20.491 & 24.954 & 20.681 & 24.456 & 18.368 & 22.364 \\
20 & 1500 & 12.765 & 15.281 & 12.565 & 15.396 & 14.069 & 16.957 \\
\hline
\end{tabular}

The comparison between figure 4 and figure 6 shows that for the prediction of algal cell density, the predicted $R^{2}$ value of ga-bp neural network is about $13 \%$ higher than the predicted $R^{2}$ value of BP network. $100 \%$ of the ga-bp predictions had an error of less than $10 \%$, and only $40 \%$ of the BP predictions had an error of less than $10 \%$. As can be seen from figure 5 and figure 7 , for the prediction of the mean radius of algal cells, the predicted $R^{2}$ value of ga-bp neural network is about $10 \%$ higher than the predicted $R^{2}$ value of BP network. The $100 \%$ ga-bp prediction error is less than $10 \%$. Only $70 \%$ of BP forecasts are off by less than $10 \%$. By comparison, the prediction accuracy of ga-bp algorithm is obviously higher than that of the traditional BP neural network. 


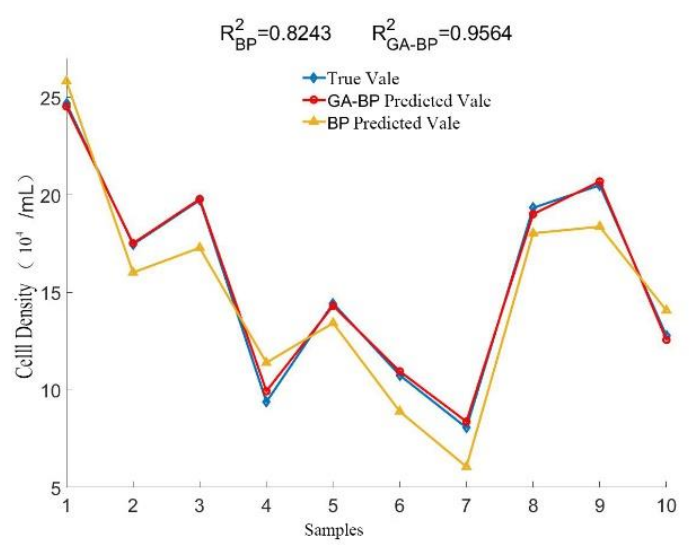

Fig. 4: Comparison of measured and predicted results of algal cell density

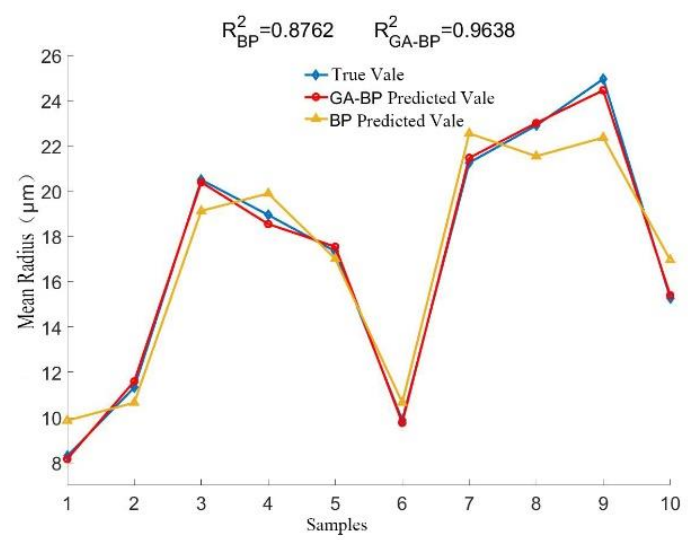

Fig. 5: Comparison of measured values and predicted results of the average algal cell radius

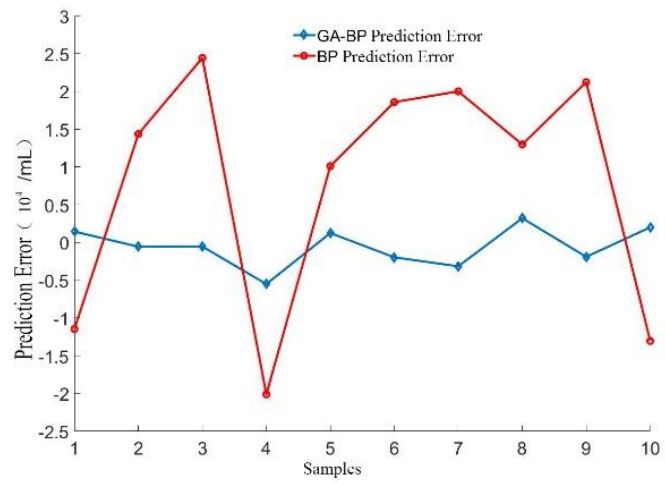

Fig. 6: Comparison chart of algal cell density prediction error

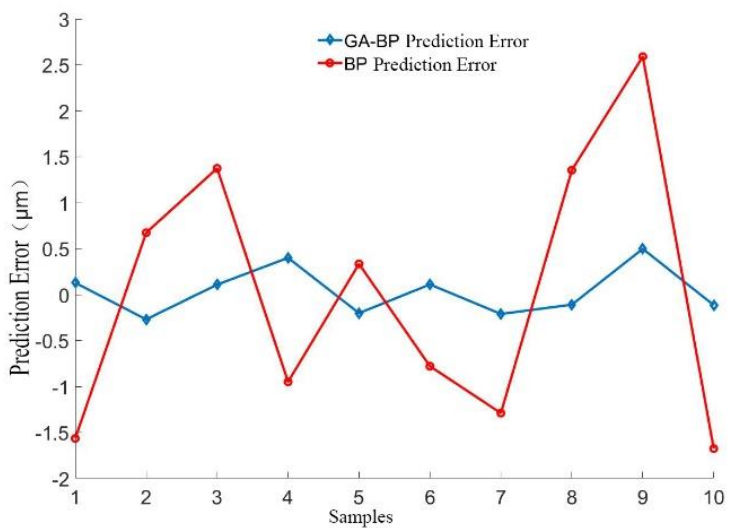

Fig. 7: Comparison of prediction errors of the average algal cell radius 


\section{Conclusions}

In this study, the growth prediction model of BP neural network was constructed by using the light intensity and temperature of the growth of cymbidium aspergillus as the input of BP neural network, and the density and average radius of algal cells as the output, and the weight and threshold of BP neural network were optimized by genetic algorithm. It is verified that the optimized BP neural network has higher prediction accuracy. At the same time, it also provides a new idea for the research of growth prediction model of microalgae.

\section{Acknowledgments}

This work was supported by the National Key Research and Development Program of China (Grant No.2017YFB0403904) and This work was supported by Tianjin Key Laboratory of information sensing and intelligent control.

\section{References}

[1] Demirbas, A.D.M.F. Algae Energy Algae as a New Source of Biodiesel [M]. London: Springer, 2010: 75-99.

[2] Carvalho, A.P., L.A. Meireles, et al. Microalgal Reactors: A Review of Enclosed System Designs and Performances [J]. Biotechnology Progress, 2006, 22(6): 1490-1506.

[3] Garcia-Casal MN, Ramirez J, Leets I, et al. Antioxidant capacity, polyphenol content and iron bioavailability from algae (Ulva sp.,Sargassum sp. and Porphyra sp.) in human subjects. British Journal of Nutrition,2009, 101:79-85.

[4] Harun, R., et al. Bioprocess engineering of microalgae to produce a variety of consumer products [J]. Renewable and Sustainable Energy Reviews, 2010, 14(3): 1037-1047.

[5] Romera E, Gonzalez F, Balleste A, et al. Biosorption of $\mathrm{Cd}, \mathrm{Ni}$, and $\mathrm{Zn}$ with mixtures of different types of algae[J]. Environmental Engineering Science, 2008, 25:999-1008.

[6] Shi J, Podola B, Melkonian M. Removal of nitrogen and phosphorus from waste water using microalgae immobilized on twin layers: an experimental study[J]. Journal of Applied Phycology , 2007, 19:417-423.

[7] Luo zhizhan etc.. Advances in the treatment of wastewater by microalgae[J]. Water treatment technology, 2019, 45(10): page17-23+39.

[8] Lee, E., M. Jalalizadeh, et al. Growth kinetic models for microalgae cultivation: A review [J]. Algal Research, 2015, 12: 497-512.

[9] Han BP, Virtanen M, Koponen J, Straškraba M. Effect of photoinhibition on algal photosynthesis: a dynamic model[J]. J Plankton Res, 2000, 22:865-885.

[10] Moheimani NR. Inorganic carbon and $\mathrm{pH}$ effect on growth and lipid productivity of Tetraselmis suecica and Chlorella sp (Chlorophyta) grown outdoors in bag photobioreactors[J]. J Appl Phycol, 2013, 25:387-398.

[11] J.C. Goldman. Outdoor algal mass cultures -II. Photosynthetic yield limitations[J]. Water Res, 13 (1979) 119-136.

[12] Darvehei, P., P.A. Bahri an, N.R. Moheimani. Model development for the growth of microalgae: A review[J]. Renewable and Sustainable Energy Reviews. 2018, 97: 233-258.

[13] Gao wenfeng, Yao zhihong.Prediction of algal growth based on BP neural network[J]. Microcomputer information.2005, (18): 167-169.

[14] Wang qi, Qiao hongyu. Prediction of microbial quantity in ballast water based on ga-bp neural network[J]. Journal of jiangsu university of science and technology (natural science edition). 2016, 30(06): 529-537.

[15] Bernard O, Rémond B. Validation of a simple model accounting for light and temperature effect on microalgal growth[J]. Bioresour Technol . 2012, 123: 520-527.

[16] Orosa M, Franqueira D, Cid A, et al. Analysis and enhancement of astaxanthin accumulation in Haematococcus pluvialis[J]. Bioresource Technol. 2005: 373-378.

[17] Aflalo, Zarka, Boussiba, On the relative efficiency of two-vs. onestage production of astaxanthin by the green alga Haematococcus pluvialis[J]. Biotechnol. Bioeng. 2007: 300-305. 
[18] He zhengfeng. MATLAB R2015b Neural network technique[M]. Beijing:Tsinghua university press, 2016: 156205.

[19] Lei yingjie, Zhang shanwen, Li xuwu, etc.. Genetic algorithm toolbox and its application[M]. Xian: Xidian university press, 2005: 11-31.

[20] Yang huimin. Study on Culture Conditions of High Astaxanthin from Haematococcus pluvialis[D]. Guangdong ocean university, 2016: 10-40. 